\title{
Microbiologie et maladies infectieuses
}

\section{Philippe Sansonetti}

\section{OpenEdition}

\section{Journals}

Édition électronique

URL : https://journals.openedition.org/annuaire-cdf/15616

DOI : 10.4000/annuaire-cdf.15616

ISBN : 978-2-7226-0572-5

ISSN : 2109-9227

Éditeur

Collège de France

Édition imprimée

Date de publication : 30 décembre 2020

Pagination : 151-156

ISBN : 978-2-7226-0516-9

ISSN : 0069-5580

Référence électronique

Philippe Sansonetti, « Microbiologie et maladies infectieuses », L'annuaire du Collège de France [En ligne], 118 | 2020, mis en ligne le 01 avril 2021, consulté le 22 août 2022. URL : http://

journals.openedition.org/annuaire-cdf/15616 ; DOI : https://doi.org/10.4000/annuaire-cdf.15616 


\title{
MICROBIOLOGIE ET MALADIES INFECTIEUSES
}

\author{
Philippe SANSONETTI \\ Membre de l'Institut (Académie des sciences), \\ professeur au Collège de France
}

Mots-clés : bactéries, microbiote, maladies infectieuses, virus

La série de cours et séminaires "Symbiose, antibiose, eubiose, dysbiose : quand les bactéries osent » est disponible, en audio et/ou en vidéo, sur le site internet du Collège de France (https://www.college-de-france.fr/site/philippe-sansonetti/ course-2017-2018.htm), ainsi que le colloque "Microbiome and human evolution: (Micro)Biology meets anthropology » (https://www.college-de-france.fr/site/philippesansonetti/symposium-2017-2018.htm).

\section{ENSEIGNEMENT}

COURS ET SÉMINAIRES - SYMBIOSE, ANTIBIOSE, EUBIOSE, DYSBIOSE : QUAND LES BACTÉRIES OSENT

Le cours 2017-2018 a été un point d'étape important dans un domaine extrêmement dynamique de la microbiologie : le microbiote. L'objectif était une mise à jour sur des sujets clés: les mécanismes assurant stabilité, robustesse et résilience du microbiote intestinal ainsi que les mécanismes sous-tendant son effet de barrière contre des microbes intrus, en particulier pathogènes ; les bases et la rationalité des symbioses étudiées dans des systèmes modèles animaux, avec un intérêt particulier pour le rôle de l'interaction microbe/hôte dans le développement de ce dernier, les «fables de symbioses ». Nous nous sommes interrogés sur l'existence de symbioses virales véritables aux confins de la latence et leur rôle possible, en particulier dans la maturation du système immunitaire, voire de sa sénescence et de la survenue de maladies auto-immunes. Nous avons également étudié les moyens de passer de corrélation à causalité dans les effets pathologiques associés à un déséquilibre du microbiote (dysbiose) et avons tenté d'établir une définition des signatures microbiologiques pathologiques et d'adapter à des populations microbiennes 
complexes des «postulats de Koch revisités ». Nous nous sommes enfin intéressés à l'influence développementale réciproque de divers microbiotes et du nouveau-né/ nourrisson.

Chacun des cours a été précisé par un séminaire mettant en avant et développant un sujet particulier en rapport avec le thème du cours, donné par un éminent spécialiste du domaine considéré. Ces séminaires, au-delà de la thématique qu'ils traitaient, insistaient sur les approches et méthodologies expérimentales utilisées au laboratoire ou sur le terrain.

\section{Cours 1 - Effet de barrière du microbiote : symbiose et antibiose}

Ce cours a envisagé les éléments assurant la propriété de résistance à la colonisation du microbiote intestinal, effet considérable pouvant, dans des modèles animaux, nécessiter une augmentation de plusieurs ordres de grandeur de la taille de l'inoculum pour assurer le succès d'une infection par rapport à un animal dénué de microbiote. Cet effet de barrière est dû à la conjonction d'un réseau métabolique stable établi par la flore résidente et privant l'intrus des nutriments nécessaires à sa croissance ainsi qu'à la production de molécules et de systèmes bactéricides tels les colicines et les appareils de sécrétion de type 6 capables de perforer les membranes bactériennes et pour les seconds d'injecter des enzymes et toxines dans le cytoplasme des bactéries cibles. Le rôle précis des bactériophages dans le maintien de ces équilibres reste à démontrer.

\section{Séminaire 1 - Guerres intestines : le système de sécrétion de type 6 , un atout dans la course aux armements bactérienne}

Éric Cascales (CNRS, Marseille)

Ce séminaire a magnifiquement illustré la façon dont la conjonction de la génétique bactérienne, de l'imagerie dynamique et de la biologie structurale permet de déchiffrer le mode d'action et la régulation du fonctionnement de l'appareil bactérien de sécrétion de type 6 .

\section{Cours 2 - Fable de symbioses : le calamar et la fourmi}

Ce cours a envisagé, au-delà de la description des microbiotes de ces organismes, comment des bactéries ou populations microbiennes peuvent affecter le développement et la fonction d'un organe de l'hôte, comme le rôle de Vibrio fischeri dans la genèse et la fonction de l'organe lumineux du calamar et la conjonction de certaines bactéries et d'un mycétome fongique dans le comportement communautaire des fourmis attiques jardinières.

\section{Séminaire 2 - Symbioses végétales. La plante, entre holobionte et réseaux d'interactions}

Marc-André Sélosse (Muséum national d'histoire naturelle, Paris)

Ce séminaire a offert une belle réflexion sur la symbiose bactéries/plantes et sur la validité ou non du concept d'holobionte chez les plantes, considérant que des plantes différentes peuvent être amenées à partager la même rhizosphère. 


\section{Cours 3 - Fables de symbioses (2) : l'abeille et le moustique}

Dans la même ligne que la précédente leçon, au-delà de la description du microbiote intestinal de ces deux insectes, nous avons envisagé leur rôle développemental, trophique et de protection contre les maladies infectieuses et parasitaires. Ces travaux ouvrent des pistes de protection des abeilles, espèce actuellement en danger, et de possibles solutions pour le contrôle des populations vectorielles d'arthropodes transmettant des infections virales et parasitaires.

\section{Séminaire 3 - Que nous apprennent les modèles animaux gnotobiotiques sur le rôle du microbiote intestinal dans le développement et le métabolisme?}

François Leulier (CNRS, Lyon)

Ce séminaire a fait le point sur un magnifique travail de groupe ayant permis de démontrer le rôle essentiel du microbiote intestinal dans le développement, le trophisme et la nutrition de la mouche Drosophila melanogaster. Ces aspects peuvent largement être phénocopiés par une seule souche comme Lactobacillus plantarum, offrant la possibilité d'une approche génétique visant à identifier les effecteurs microbiens influençant le cycle développemental de la mouche et au-delà, si l'on considère que l'évolution a largement « recyclé » les bonnes solutions.

\section{Cours 4 - Des symbioses virales ?}

Ce cours avait pour but de mieux définir les contours de ce qui pourrait être un véritable virome humain, c'est-à-dire une collection d'espèces virales réellement engagées dans une symbiose mutualiste à différents niveaux, mais particulièrement dans la peau, l'intestin et le «milieu intérieur». Cela semble être le cas des anellovirus acquis très tôt dans la vie. Il est cependant difficile à ce stade de définir si l'on se situe dans une véritable situation de symbiose mutualiste, ou aux confins de la latence virale. Ce sujet est particulièrement épineux avec les virus Herpès dont la latence n'est pas synonyme de quiescence totale comme le montrent les études transcriptomiques. Il n'est pas impossible qu'en bloc les virus Herpès jouent un rôle dans la maturation du système immunitaire du sujet jeune et la protection contre les infections, puis un rôle délétère dans la sénescence du système immunitaire chez les personnes âgées.

\section{Séminaire 4 - Exploration du virome humain, de la symbiose à la pathologie}

Christelle Desnues (CNRS, Marseille)

Ce séminaire a très bien illustré comment les méthodes de séquençage de nouvelle génération permettent d'explorer le virome humain, qu'il s'agisse de bactériophages ou de virus eucaryotes.

\section{Cours 5 - Eubiose et dysbiose, où sont les signatures ?}

Ce cours fut l'occasion de revisiter les «postulats de Koch» à l'aune des populations microbiennes complexes dont les altérations qualitatives et quantitatives, ou dysbioses, sont associées à des pathologies. Est-il possible, afin d'établir un lien 
de causalité, d'appliquer inchangés les « postulats de Koch » qui ont été établis pour un microorganisme pathogène unique et non pour une population microbienne plus ou moins diverse ? Nous proposons la notion de «postulats écologiques de Koch» et tentons de faire émerger des critères établissant au plus près la causalité.

\section{Séminaire $\mathbf{5}$ - Le poids du microbiote dans l'obésité et le diabète}

Karine Clément (université Pierre-et-Marie-Curie et hôpital de la Pitié-Salpétrière)

Ce séminaire a illustré comment les études cliniques dans le domaine de l'interface entre dysbiose intestinale et métabolisme permettent de faire émerger des associations corrélatives avec des pathologies tels l'obésité, le diabète, la cirrhose hépatique et le NASH. Ce séminaire a aussi permis de percevoir comment les études cliniques préparent des études interventionnelles qui pourront certes améliorer les pathologies mentionnées, mais aussi confirmer les liens de causalité. Superbe travail «du laboratoire au lit du patient et retour».

\section{Cours 6 - Des microbes et des petits d'hommes : hasard et nécessité de l'holobiose}

Ce cours a passé en revue les grandes étapes du développement de l'enfant depuis la conception jusqu'à la deuxième année de vie, soit les « mille premiers jours ». Il a évoqué la possibilité d'un microbiote placentaire et méconial qui pourrait avoir un rôle dans le développement du fœus, en particulier la maturation anténatale de son système immunitaire. Il a montré les premiers éléments disponibles sur la construction du microbiote du nouveau-né et les risques de son altération par des manœuvres comme la césarienne qui « court-circuite » la possibilité de colonisation précoce du tube digestif de l'enfant par la flore maternelle vaginale et fécale ou la prise importante d'antibiotiques. Ces situations «post-modernes » appauvrissent le microbiote de l'enfant et semblent en mesure de précipiter la survenue de maladies en croissance inquiétante comme l'asthme, l'allergie et l'atopie, l'obésité et le diabète. C'est un champ nouveau de la médecine qui s'ouvre et mérite attention car des interventions précoces d'enrichissement et de diversification du microbiote intestinal pourraient contribuer à prévenir ces pathologies.

\section{Séminaire 6 - Entéropathie environnementale pédiatrique : de la dysbiose à la malnutrition}

Pascale Vonaesch (Institut Pasteur, Paris)

Ce séminaire a développé les caractéristiques d'un syndrome affectant l'intestin grêle de par la présence d'une dysbiose massive dans le duodéno-jéjunum. Cette entéropathie, responsable d'une malnutrition, cause un retard de croissance accompagné d'un retard de développement psychomoteur. La physiopathologie de ce syndrome est mal connue, donc les interventions préventives et thérapeutiques pour l'instant limitées. Ce séminaire présente une étude coordonnée par notre laboratoire en Afrique subsaharienne et à Madagascar où la maladie est très prévalente. Cette étude a permis d'identifier la composition de la dysbiose duodénale de ces enfants et de montrer qu'elle était dominée, de manière totalement inattendue, par une flore buccale et pharyngée, changeant totalement le paradigme physiopathologique et offrant des solutions de prévention. 


\section{COLLOQUE INTERNATIONAL - MICROBIOME AND HUMAN EVOLUTION: (MICRO)BIOLOGY MEETS ANTHROPOLOGY}

Ce symposium international organisé par Philippe Sansonetti (Collège de France) et Brett Finlay (université de Colombie-Britannique, Canada) s'est déroulé les 28 et 29 juin 2018 au Collège de France. Il a été soutenu par la Fondation Hugot du Collège de France, le Peter Wall Institute for Advanced Studies (UBC, Canada) et le Canadian Institute for Advanced Research (Canada).

L'objectif était de réunir médecins, biologistes et anthropologues autour du thème de la relation entre le microbiome et le développement humain. Seize orateurs internationaux ont donc été réunis lors de ce colloque, qui s'est conclu par une réunion sous forme de "brainstorming » afin d'approfondir la réflexion sur les grandes lignes à développer à cette interface.

Les grands axes des présentations et de la réflexion ont été :

- séquençage de l'ADN fossile et contemporain et développement de modèles d'interaction hôte-microbes afin d'étudier l'impact du microbiote sur l'évolution de l'espèce humaine ;

- développements de la socio-anthropologie et leur impact sur l'analyse épidémiologique des situations d'interface homme-microbes. Vers une épidémiologie qualitative ;

- comment les microbes affectent des fonctions biologiques qui furent essentielles dans l'évolution de l'espèce humaine; recherche de situations contemporaines, de leurs déterminants et développement de modèles.

\section{PuBlications}

Anderson M.C., Chaze T., CoḮ Y.-M., Injarabian L., Jonsson F., Lombion N., Selimoglu-Buet D., Souphron J., Ridley C., Vonaesch P., Baron B., Arena E.T., TineVeZ J.-Y., Nigro G., Nothelfer K., Solary E., Lapierre V., LAZure T., Matondo M., ThORNTON D., SANSONETTI P.J., BALEUX F. et MARTEYN B.S., « MUB40 binds to lactoferrin and stands as a specific neutrophil marker », Cell Chemical Biology, vol. 25, ${ }^{\circ}$ 4, 2018, p. 483-493.e9, DOI : 10.1016/j.chembiol.2018.01.014.

Aymeric L., Donnadieu F., Mulet C., Du Merle L., Nigro G., Saffarian A., Bérard M., Poyart C., Robine S., Regnault B., Trieu-Cuot P., Sansonetti P.J. et Dramsi S., "Colorectal cancer specific conditions promote Streptococcus gallolyticus gut colonization ", Proceedings of the National Academy of Sciences of the United States of America, vol. 115, $\mathrm{n}^{\mathrm{o}}$ 2, 2018, E283-E291, DOI : 10.1073/pnas.1715112115.

Belotserkovsky I., Brunner K., Pinaud L., Rouvinski A., Dellarole M., Baron B., Dubey G., Samassa F., Parsot C., Sansonetti P. et Phalipon A., "Glycan-glycan interaction determines Shigella tropism toward human T lymphocytes », MBio, vol. 9, $\mathrm{n}^{\circ} 1$, 2018, 02309-17, DOI : 10.1128/mBio.02309-17.

Charlier P., Augias A., Sansonetti P., Bon C., Kennedy S. et Segurel L., « Faces vivos docent: Microbiome intestinal ancien et problématiques médicales contemporaines = Importance of intestinal paleomicrobiome study for contemporaneous medical problematics », Médecine/sciences, vol. 33, nº 11, 2017, p. 984-990, DOI : 10.1051/medsci/20173311016.

Charlier P., Augias A., Sansonetti P., Bouchet F., Bon C., Kennedy S. et Segurel L., «Étude paléoparasitologique des latrines du Pavillon royal et du $3^{\mathrm{e}}$ pavillon du Levant du château de Marly », Bulletin du Centre de recherche du château de Versailles. Sociétés de cour en Europe, XVI ${ }^{e}$-XIX ${ }^{e}$ siècle, 2017, DOI : 10.4000/crcv.14092. 
Corelli B., Almeida A.S., Sonego F., Castiglia V., Fevre C., Brisse S., Sansonetti P.J. et TournebizE R., «Rhinoscleroma pathogenesis: The type K3 capsule of Klebsiella rhinoscleromatis is a virulence factor not involved in Mikulicz cells formation », PLoS Neglected Tropical Diseases, vol. 12, no 1, 2018, e0006201, DOI : 10.1371/journal. pntd.0006201.

Lapaquette P., Fritah S., Lhocine N., AndrieuX A., Nigro G., Mounier J., SANSOnetti P. et DEJEAN A., « Shigella entry unveils a calcium/calpain-dependent mechanism for inhibiting sumoylation », ELife, vol. 6, 2017, e27444, DOI : 10.7554/eLife.27444.

Naito T., Mulet C., De Castro C., Molinaro A., Saffarian A., Nigro G., Bérard M., Clerc M., PEDERSEN A.B., SANSONETTI P.J. et PÉDRON T., « Lipopolysaccharide from cryptspecific core microbiota modulates the colonic epithelial proliferation-to-differentiation balance », MBio, vol. 8, no 5, 2017, DOI : 10.1128/mBio.01680-17.

Pinaud L., Ferrari M.L., Friedman R., Jehmlich N., von Bergen M., Phalipon A., SANSONETTI P.J. et CAMPBELL-VALOIS F.-X., « Identification of novel substrates of Shigella T3SA through analysis of its virulence plasmid-encoded secretome », PloS One, vol. 12, $\mathrm{n}^{\circ} 10$, 2017, e0186920, DOI : 10.1371/journal.pone.0186920.

Pinaud L., Samassa F., Porat Z., Ferrari M.L., Belotserkovsky I., Parsot C., SANSONETTI P.J., CAMPBEll-VAlOIS F.-X. et PHALIPON A., «Injection of T3SS effectors not resulting in invasion is the main targeting mechanism of Shigella toward human lymphocytes », Proceedings of the National Academy of Sciences of the United States of America, vol. 114, no 37, 2017, p. 201707098, DOI : 10.1073/pnas.1707098114.

Pinaud L., SAnsonetTi P.J. et Phalipon A., «Host cell targeting by enteropathogenic bacteria T3SS effectors », Trends in Microbiology, vol. 26, $\mathrm{n}^{\circ}$ 4, 2018, p. 266-283, DOI : 10.1016/j.tim.2018.01.010.

SANSONETTI P., « La défiance vis-à-vis des vaccins, un luxe pour ceux qui sont protégés par les autres ? », Après-demain, vol. 42, nº 2, 2017, p. 9-11.

SANSONETTI P.J., «Editorial for the virtual issue on microbiome », FEMS Microbiology Reviews, vol. 42, n 2, 2018, p. 113-115, DOI : 10.1093/femsre/fux058.

SANSONETTI P.J., « Measles 2018: A tale of two anniversaries », EMBO Molecular Medicine, vol. 10, nº 5, 2018, e9176, DOI : 10.15252/emmm.201809176.

Vonaesch P., Anderson M. et SANSONETti P.J., « Pathogens, microbiome and the host: Emergence of the ecological Koch's postulates », FEMS Microbiology Reviews, vol. 42, $\mathrm{n}^{\circ} 3$, 2018, p. 273-292, DOI : 10.1093/femsre/fuy003.

VONAESCH P., SANSONETTI P.J. et SCHNUPF P., «Immunofluorescence analysis of stress granule formation after bacterial challenge of mammalian cells », Journal of Visualized Experiments, $\mathrm{n}^{\mathrm{O}}$ 125, 2017, e55536-e55536, DOI : 10.3791/55536.

Vonaesch P., Tondeur L., Breurec S., Bata P., Nguyen L.B.L., Frank T., Farra A., RAFAÏ C., Giles-Vernick T., Gody J.C., GouAndJIKA-VAsilache I., SANSONETTI P. et VRAY M., «Factors associated with stunting in healthy children aged 5 years and less living in Bangui (RCA)», PloS One, vol. 12, no 8, 2017, e0182363, DOI : 10.1371/journal. pone.0182363.

Xu D., Liao C., Zhang B., Tolbert W.D., He W., Dai Z., Zhang W., Yuan W., Pazgier M., LiU J., Yu J., SANSONETti P.J., BeVInS C.L., ShaO Y. et LU W., « Human enteric $\alpha$-defensin 5 promotes Shigella infection by enhancing bacterial adhesion and invasion », Immunity, vol. 48, $n^{\circ} 6,2018$, p. 1233-1244.e7, DOI : 10.1016/j.immuni.2018.04.014. 